\title{
ECONOMICAL IMPACT OF ORCHIECTOMY FOR ADVANCED PROSTATE CANCER
}

\author{
ADRIANO A. P. DE PAULA, HILTON R. S. PICCELLI, NILSON P. PINTO, ANTONIO G. \\ TELES, ANTONIO G. FRANQUEIRO, ADRIANO R. L. MALTEZ, JOSE H. SILVA \\ Section of Oncological Urology, Araujo Jorge Hospital, Association Against Cancer of \\ Goias (ACCG), Goiania, Góias, Brazil
}

\begin{abstract}
Purpose: To demonstrate the economical impact of surgical castration in comparison to the medical castration for patients with advanced prostate cancer.

Material and Methods: Between January 2001 and December 2001, 32 patients with advanced prostate cancer underwent bilateral sub-capsular orchiectomy at our Hospital. The costs of this procedure were compared to the costs of medical castration with LH-RH analogues.

Results: The costs of the surgical procedure were extremely reduced when compared to published data on the medical treatment. Surgical castration did not have any stronger negative impact on the evolution of these patients when compared to medical castration.

Conclusion: Surgical castration is an efficient and low cost treatment for advanced prostate cancer.
\end{abstract}

Key words: prostate; prostatic neoplasms; castration; therapy; costs and cost analysis; androgen antagonists

Int Braz J Urol. 2003; 29: 127-32

\section{INTRODUCTION}

Most prostate cancers are hormone-dependent; this means that they need testosterone for disease progression. Ninety percent of testosterone is produced by Leydig cells of the testis, whereas adrenal glands account for 5\% of this production. Survival after treatment can reach 10 to 15 years as shown by Johansson \& Ljunggren (1981).

The simplest way to get a hormonal blockade is bilateral orchiectomy, a procedure described by Huggins \& Hodges (1). Riba (1942) modified the original technique to avoid an empty scrotum and described the subcapsular bilateral orchiectomy. The advantages of this technique are shown in Table-1.

The most popular treatment to reach hormonal blockade is the use of LH-RH analogues. The greatest problem of this modality of treatment is its high cost, especially if there is an expectation of ex- tended survival, as is currently observed in the literature. Other disadvantages are shown in Table 2 (2).

Hormonal blockade can also be achieved by using estrogens, steroidal anti-androgens and non-steroidal anti-androgens, but none of these methods is more effective or has fewer side effects than surgical castration $(3,4)$. Not even maximal blockade has shown better results when compared to orchiectomy alone or to the single use of LH-RH analogues (only 3 out of 27 randomized trials showed the benefits of maximal blockade) $(5,6)$.

Great savings can be achieved when LH-RH analogues are replaced by bilateral orchiectomy, even when the latter is adopted after hormone refractory disease.

\section{MATERIALS AND METHODS}

Thirty-two patients with advanced prostate cancer underwent bilateral orchiectomy at our Hos- 
Table 1-Advantages of surgical castration for advanced prostate cancer.

- Low cost procedure (local anesthesia, out patient)

- Immediate effect (castrate levels of testosterone in 12h)

- Low morbidity

- Effectiveness comparable to other clinical castration methods

- Definitive treatment

- No psychological negative effects (no empty scrotum)

- No need of anti-androgens to avoid "flare"

pital during the year of 2001, and their clinical and biochemical evolution, as well as the costs of the treatment, were analyzed.

All patients had T3, T4 or any T adenocarcinoma of the prostate with bone metastasis.

Bilateral orchiectomy was indicated to these patients, in view of the need for continuous treatment. It was adopted as primary procedure or as replacement of another hormonal blockade. All patients were informed on the details of the surgical procedure, its risks and its possible complications.

The operation was performed under local anesthesia and sedation, and there were no in-patients. The mean time of the procedure was 17 minutes, and $100 \mathrm{mg}$ i.v. of cetoprofen and $1 \mathrm{~g}$ i.v. cefazolin were routinely administrated.

Data on patients and their biochemical evolution are shown in Table-3. The mean age of the patients was 73.81, ranging from 59 to 93 . Eighteen patients had a Gleason score of up to 6 , and 5 presented figures above 7 .

Table 2 - Disadvantages of LH-RH analogues for advanced prostate cancer.

- Continuous or periodic treatment (long-life)

- Negative psychological effects due to the continuous treatment

- High costs to public and private health care companies

- Risk of non-effective medication if not well stored

- Need of anti-androgens to avoid "flare"

- High cost
The "Unified Health System", which comprises public health-care in Brazil, covered the costs of all patients.

\section{RESULTS}

None of the patients stayed in hospital for more than 12 hours on the day of surgery.

The median follow up was of 11.43 months.

Eight patients had taken anti-androgens before the procedure. In this work only 4 patients required anti-androgens after surgery to control rising PSA, whereas the PSA levels of 27 of them decreased after the intervention. The lowest nadir obtained was $0.08 \mathrm{ng} / \mathrm{mL}$.

Four patients showed no PSA decrease 30 days after orchiectomy, but 3 of them had already been diagnosed for hormone refractory disease.

Two patients died of prostate cancer, respectively 5 and 9 months after surgery (at their first presentation, the former patient had a PSA level of 890 $\mathrm{ng} / \mathrm{mL}$ and the latter had already been restricted to bed due to bone metastasis at lumbar spine).

No patient required any medication other than cetoprofen and paracetamol to control surgical pain.

Three patients presented scrotal hematoma and were treated with antibiotics and local care.

No psychological complaints were referred to the surgery.

\section{DISCUSSION}

The amount paid by the Brazilian public health care for each bilateral orchiectomy was US\$ 43. The cost of surgical castration varies depending on the country where it is performed and considering different kinds of social security systems.

In the present context, a single dose of LH$\mathrm{RH}$ analogue costs 1.6 times more when compared to the surgical procedure. Considering the median follow up of 11.41 months and the fact that those 30 patients remained alive at the end of this work, we can estimate a total cost of US\$33,387.63 with LHRH analogues.

The subcapsular technique bypasses the need for prosthesis thus contributing to a lower cost when 
Table 3 - Data of the patients.

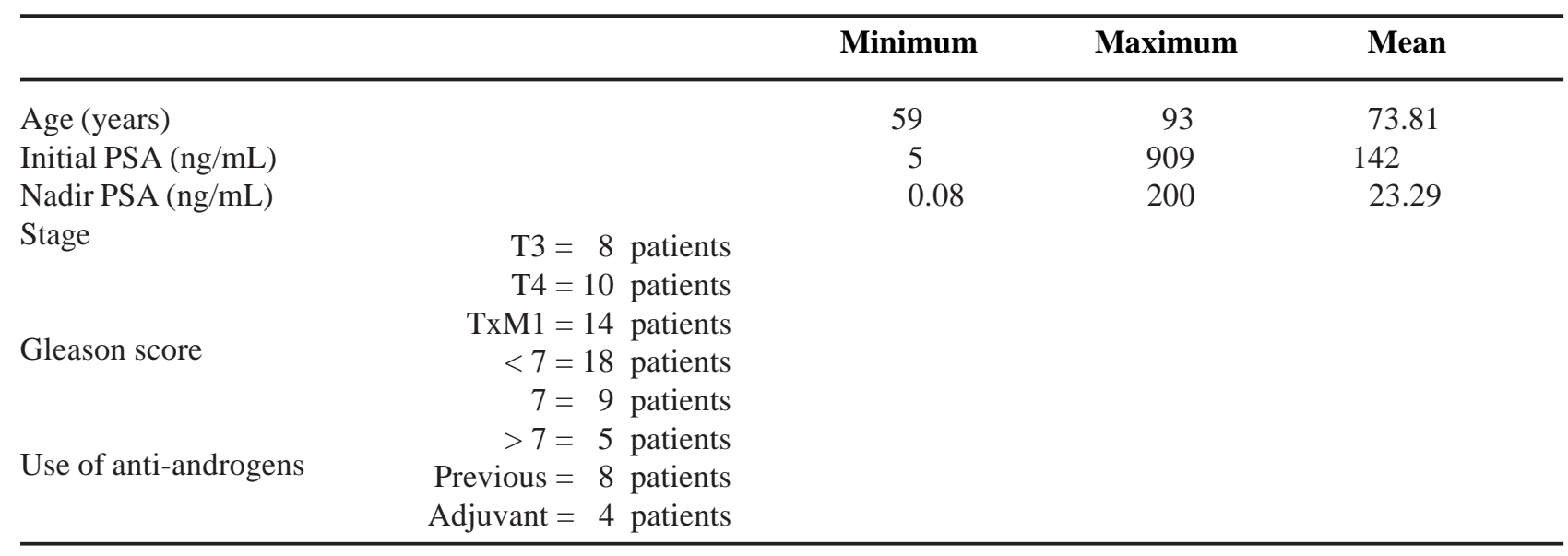

compared to total orchiectomy. Chapman reported no difference on testosterone and $\mathrm{LH}$ values when comparing subcapsular to total orchiectomy (7). Hering et al. also showed no statistical difference between total and subcapsular orchiectomy, nevertheless, related $43 \%$ of psychological side effects in the subcapsular group (8).

Side effects like mastalgy, gastric symptoms and higher risk of deep vein thrombosis were not seen in our work, but they are reported when estrogens or anti-androgens are used $(3,4)$.

Public health entities can reap significant savings when castration is taken as a treatment for advanced prostate cancer. Depending on the country, a single dose of LH-RH analogue can be more expensive than bilateral orchiectomy.

This paper clearly shows that surgical castration, at our institution, is an efficient, inexpensive, simple and low psychological effect modality of treatment for advanced prostate cancer. In addition, the quality of life of patients submitted to orchiectomy was good, as has already been shown by several papers (11). This work shows that, if well informed, patients with advanced prostate cancer accept bilateral orchiectomy well.

Better results in relation to nadir could have been achieved with a longer follow up, but this is not the aim of the present work (10).

There will always be indication for other hormone blockade modalities (mainly LH-RH ana- logues) in situations such as intermittent treatment, radiotherapy adjuvant or concomitant treatment or even in cases in which the patient does not accept surgical castration (9).

Mariani \& Glover suggested that the savings brought about by the substitution of LH-RH analogues for surgical castration should be channeled to research of new prostate cancer treatment modalities (12).

\section{CONCLUSIONS}

This work shows that bilateral subcapsular orchiectomy is a low cost, efficient modality of treatment for advanced prostate cancer at our institution. Its acceptance is quite good and its psychological effects are almost inexistent.

We suggest the urologist who deals with prostate cancer to consider the economical impact when offering the different ways to treat advanced prostate cancer, or, at least, to propose surgical castration when the diagnosis of hormone refractory disease is made.

\section{REFERENCES}

1. Huggins C, Hodges CV: Studies on prostatic cancer: the effect of castration of estrogen and of androgen injection on serum phosphatases in metastatic carcinoma of the prostate. Cancer Res. 1941; 1: 293-7.

2. Stricker HJ: Luteinizing hormone-releasing hormone antagonists in prostate cancer. Urology 2001; 58: 24-7. 
3. Kolvenbag GJ, Iversen P: Anti-androgen monotherapy: a new form of treatment for patients with prostate cancer. Urology 2001; 58: 16-23.

4. Malkowics SB: The role of Diethylstilbestrol in the treatment of prostate cancer. Urology 2001; 58: 108-13.

5. Collette L, Studer UE: Why phase III trials of maximal androgen blockade versus castration in M1 prostate cancer rarely shows statistically significant differences. Prostate 2001; 48: 29-39.

6. Schmitt B, Witt TJ: Combined androgen blockade with non-steroidal Anti-androgens for advanced prostate cancer: a systematic review. Urology 2001; 57: 727 32.

7. Chapman JP: Comparison of testosterone and LH values in subcapsular vs total orchiectomy patients. Urology 1987; 30: 27-8.

8. Hering FL, Dall'oglio MF, Caponero R, Rodrigues PR, Nesrallah LJ, Srougi M: Total versus subcapsular orchiectomy for treatment of advanced prostatic carcinoma: Comparison of serum testosterone and PSA levels. J Bras Urol. 1999; 25: 221-4 [in Portuguese].

9. Bolla M, Gonzalez D, Warde P, Dubois JB, Mirimanoff RO, Storme G, et al.: Improved survival in patients with locally advanced prostate cancer treated with radiotherapy and goserelein. N Engl J Med. 1997; 337: 295-300.

10. Benaim EA, Pace CM: Nadir prostate-specific antigen as a predictor to progression to androgen-independent prostate cancer. Urology 2002; 59: 73-8.

11. Nygard R, Norum J: Goserelin (Zoladex) or orchiectomy in metastatic Prostate cancer? A quality of life and cost-effectiveness analysis. Anticancer Res. 2001; 21: 781-8.

12. Mariani AJ, Glover M: Medical versus surgical androgen suppression therapy for prostate cancer: a 10-year longitudinal cost study. J Urol. 2001; 165: 104-7.
Received: November 8,2002 Accepted after revision: February 17, 2003
Correspondence address:

Dr. Adriano A. Peclat de Paula

Hospital Araújo Jorge - Urologia

Rua 239, 181

Goiânia, GO, 74605-070, Brazil

Fax: + 5562 202-4040

E-mail: adrianopaula@hotmail.com

\section{EDITORIAL COMMENT}

The authors should be congratulated for the choice of their theme. They have the merit and the courage to discuss an infrequent issue in the literature due to obvious reactions it may induce. Although the study deals superficially with the aspect of the effectiveness of the treatment and does not allow us to draw conclusions about it, it is direct to the point about its central issue: orchiectomy is much cheaper than medical castration. In fact, the "Economical impact of orchiectomy for advanced prostate cancer" still needs more detailed studies on the types of treatment and more reflection on the part of urologists. If we consider how limited the resources of our Public Health System are, it is very important to discuss and review the amount of money that is spent on LH-RH analogues that offer the same results and benefits as an orchiectomy.

I reinforce the authors' opinion, which is certainly also shared by other urologists. Mariani, who has already been mentioned (1), explores the topic 
very well. Based upon his reflections and his data, the amounts we would spend on LH-RH analogues for just one patient of non hormone-refractory advanced disease (in an average of 30 months) would be the following: US\$ $43 \times 1.6 \times 30$ months. This means that each medical castration costs $4700 \%$ more than the US\$ 43 that are spent on an orchiectomy. Moreover, the medical procedure does not offer any logical benefit and shows the same results in terms of the patient's survival. These resources could benefit 48 patients with the definitive surgery, without taking into consideration those approximately $15 \%$ of the patients who possibly would end up migrating into the surgical treatment. In other words, for each patient treated with LH-RH analogue, we could perform an orchiectomy in one patient and still offer the access to 47 other patients to surgeries of the same type. In terms of public health, our leaders should consider these issues.

A survey held among medical doctors in the USA has indicated that $68-81 \%$ of the American urologists recommend medical anti-androgenic therapy (2). Another study has further demonstrated that when the patient is invited to choose his own treatment freely after being informed of his possibilities, $70 \%$ also prefer the medical treatment. However, when $20 \%$ of the costs of the treatment are asked for as a counter-

\section{EDITORIAL COMMENT}

Orchiectomy was the first efficient treatment for advanced prostate cancer since the work of Huggins \& Hodges, cited in the article. It is still used, which proves its value.

Many drugs have been developed to treat this disease. Each of them acts differently, but all have the same aim. However, these drugs are difficult to part on the part of the patient, only $24 \%$ choose medication (1). These figures, in addition to the fact that both treatments offer the same results, cast some doubt over the premise. They suggest the need for us to undertake studies about these types of treatment and their consequences on the patient's selfesteem, on his physical appearance and especially on his quality of life considering our socio-cultural and economical situation. An inadequate and unreal resource policy is not fair and may limit the benefit to many other patients.

\section{References}

1. Mariani AJ, Glover M: Medical versus surgical androgen suppression therapy for prostate cancer: a 10-year longitudinal cost study. J Urol. 2001; 165: 104-7.

2. Wasson JH, Fowler Jr FJ, Barry MJ: Androgen deprivation therapy for asymptomatic advanced prostate cancer in the prostate specific antigen era: a national survey of urologist beliefs and practices. $\mathbf{J}$ Urol. 1998; 159: 1993-7.

3. Iversen P, Tyrrel CJ, Kaisary AV, Anderson JB, Baert L: Casodex (bicalutamide) 150-mg monotherapy compared with castration in patients with previously untreated nonmetastatic prostate cancer: Results from two multicenter randomized trials at a median followup of 4 years. Urology 1998; 51: 389-92.

\section{Dr. Homero Arruda Division of Urology Federal University of São Paulo São Paulo, SP, Brazil}

dose and especially their price render them inaccessible to a great part of our population, which leads our patients to abandon treatment quickly and to suffer the consequences of the disease.

Among institutional patients, we notice that even with free distribution of the drug, the rate of adherence to the treatment is not as high as desired, 
which usually occurs due to the socio-economical and cultural level of these patients. Another important factor to be considered are the side-effects of many of these drugs, as well as the absolute contraindication of some drugs for patients with deep vein thrombosis.

Sub-capsular orchiectomy is an excellent option for these patients, since it is a definitive treatment, has minimal side-effects and no contraindication, and it avoids psychological trauma due to an empty scrotum.

It has been questioned whether sub-capsular orchiectomy would be as efficient as total orchiectomy. However, recent studies show that beyond being equally effective, the former is more economical, since it bypasses the need for a testicular prosthesis and disagreeable psychological side-effects (1).

This article thus demonstrates that in terms of effectiveness and costs, sub-capsular orchiectomy is extremely efficient for the treatment of advanced prostate cancer, being more economical, avoiding difficulties with doses and side-effects and keeping the esthetics of the scrotum.

\section{Reference}

1. Hering FL, Dall'oglio MF, Caponero R, Rodrigues PR, Nesrallah LJ, Srougi M: Total versus subcapsular orchiectomy for treatment of advanced prostatic carcinoma: Comparison of serum testosterone and PSA levels. J Bras Urol. 1999; 25: 221-4 [in Portuguese].

Dr. Flávio Hering Division of Urology Federal University of São Paulo São Paulo, SP, Brazil 